\title{
Z5

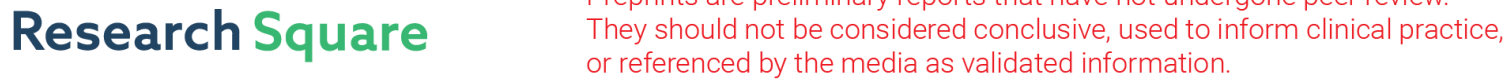 \\ Unusual Urticaria Pigmentosa in an Asian Man With Indolent Systemic Mastocytosis: A Case Report
}

\section{Chamard Wongsa}

Mahidol University Faculty of Medicine Siriraj Hospital https://orcid.org/0000-0002-5721-4881

\section{Mongkhon Sompornrattanaphan}

Mahidol University Faculty of Medicine Siriraj Hospital

\section{Torpong Thongngarm}

Mahidol University Faculty of Medicine Siriraj Hospital

\section{Weerapat Owattanapanich}

Mahidol University Faculty of Medicine Siriraj Hospital

\section{Panitta Sitthinamsuwan}

Mahidol University Faculty of Medicine Siriraj Hospital

\section{Sanya Sukpanichnant}

Mahidol University Faculty of Medicine Siriraj Hospital

Thanisorn Sukakul ( $\nabla$ kimthanisornsu@gmail.com)

Faculty of Medicine Siriraj Hospital, Mahidol University https://orcid.org/0000-0002-5351-2988

\section{Case report}

Keywords: Anaphylaxis, Indolent systemic mastocytosis, Insect sting, Mastocytosis, Urticaria pigmentosa, Maculopapular cutaneous mastocytosis

Posted Date: October 28th, 2020

DOI: https://doi.org/10.21203/rs.3.rs-96587/v1

License: (c) (i) This work is licensed under a Creative Commons Attribution 4.0 International License. Read Full License 


\section{Abstract}

Background: The diagnosis of mastocytosis remains challenging. Given that the disease has a low prevalence and its clinical presentations range from asymptomatic to severe life-threatening conditions, physicians' lack of awareness of mastocytosis is the main barrier to its diagnosis. Skin involvement is common. In adults, skin lesions are highly suggestive of systemic mastocytosis; however, clinical demonstration of lesions is difficult if they are minimal in number. In Asian, who had dark brown skin color, urticaria pigmentosa was not easy to identify. Here we present the case of indolent systemic mastocytosis with an unusual urticaria pigmentosa.

Case presentation: A 48-year-old man had had recurrent severe honeybee anaphylaxis since he was 23. He had small, subtle, brownish skin lesions on his chest and abdomen, which he and previous physicians had not recognized. The skin lesions were compatible with urticaria pigmentosa, also known as maculopapular cutaneous mastocytosis. His clinical findings and an elevated baseline tryptase level triggered a thorough systemic mastocytosis investigation. The skin and bone marrow were infiltrated by abnormal, spindle-shaped mast cells, and KIT and TET2 mutations were in the patient's serum. The honeybee anaphylaxis mechanism in this patient was IgE mediated, supported by a positive result of specific IgE to honeybee. The final diagnosis was indolent systemic mastocytosis with IgE-mediated honeybee anaphylaxis. As venom immunotherapy is unavailable in Thailand, we prescribed treatment with a regular, oral, nonsedating $\mathrm{H}_{1}$-antihistamine and an epinephrine self-injector. At the 2-year follow-up, the patient had not progressed to advanced systemic mastocytosis nor experienced any anaphylactic episodes.

Conclusion: Urticaria pigmentosa is a small, round, brown, or red maculopapular lesion. In the Asian population with dark brown skin color, physician should be exclusively careful examination, particularly in a hidden area and in anaphylaxis cases. Early recognition of urticaria pigmentosa in the adult patient might reduce the delay in diagnosis of indolent systemic mastocytosis.

\section{Background}

Mastocytosis is a rare, mast-cell, clonal disorder classified as cutaneous mastocytosis, systemic mastocytosis (SM), and mast cell sarcoma under the 2017 World Health Organization classification of tumors of hematopoietic and lymphoid tissues. ${ }^{1}$ In adult patients, SM is the most common, particularly the subvariant indolent SM (ISM). ${ }^{2}$ The prevalence of ISM in Denmark and the Netherlands is 9.6-13 per 100,000 people. $^{2}$ As epidemiological and clinical cohort studies of ISM were mainly conducted on Caucasians, Asian ISM information is scarce. We present the first case report of adult ISM in Thailand who had unusual urticaria pigmentosa, with a 23-year lag time to diagnosis.

\section{Case Presentation}


A 48-year-old, male, sugarcane farmer presented with a history of five episodes of severe honeybee anaphylaxis during a 23-year period. About 30 minutes after each honeybee sting, he felt itching at the palms before suddenly losing consciousness. On each occasion, the emergency medical staff attending to him reported that he had pronounced hypotension. For the most recent incident, initial treatment with epinephrine and hemodynamic resuscitation was provided at a primary-care hospital. He improved, and the symptoms completely resolved within a few days. He was referred to Siriraj Hospital, a tertiary-care hospital in Thailand, for further management. A physical examination was unremarkable except for multiple, discrete, ill-defined, light-brown macules on the chest and abdomen (Figure 1). Darier's sign was negative. Erythematous linear patches without edematous wheals were demonstrated when the lesions were stroked. The patient had never perceived the rash on his chest and abdomen before visiting the clinic. He denied a history of itching, flushing, abdominal discomfort, bronchospasm, fever, fatigue, and weight loss. Interestingly, he had a sister with recurrent fire ant anaphylaxis.

Skin and bone marrow biopsies confirmed a diagnosis of SM. Although the bone marrow biopsy found spindle-shaped mast cells aggregated at more than 15 cells/high power field (Figure 2), they were sparsely demonstrated in the skin biopsy (Figure 3). Blood for 25-targeted gene sequencing by a nextgeneration sequencing method (QIAseq; QIAGEN, Hilden, Germany) was positive for KIT mutation (gain of function, c.2247A>T p.D816V) and TET2 mutation (normal function, c.2440C >T p.R814C). The baseline serum tryptase was persistently high (22.4-22.6 microgram/liter; normal range < 11.4 micrograms/liter). The patient was reasonably diagnosed with ISM with skin involvement, as per the 2017 World Health Organization classification of tumors of hematopoietic and lymphoid tissues.

Blood for specific lgE (slgE) was obtained to confirm the cause of anaphylaxis. slgE to honeybee and Api m1 (Phadia ImmunoCAP) were $0.33 \mathrm{kUA} / \mathrm{L}$ and $0.16 \mathrm{kUA} / \mathrm{L}$, respectively. The total IgE was undetectable (the lower normal limit was $<4.23 \mathrm{IU} / \mathrm{ml}$ ). Although slgE was low relative to the undetectable total lgE, it was still high. Because of the strong relevant history of severe anaphylaxis after honeybee exposure, and using $0.1 \mathrm{kUA} / \mathrm{L}$ as the cutoff level for positive slgE, we diagnosed this patient as having lgE-mediated honeybee anaphylaxis.

The standard treatment for ISM with IgE-mediated honeybee anaphylaxis is lifelong venom immunotherapy. Unfortunately, the insect venom extracts are unavailable in Thailand. We prescribed regular, oral, nonsedating $\mathrm{H}_{1}$-antihistamine and an epinephrine self-injector, and prepared an anaphylaxis action plan for the patient. At the 2-year follow-up, he had not progressed to advanced SM, nor experienced any anaphylactic episodes.

\section{Discussion}

Most mastocytosis patients have skin lesions. Urticaria pigmentosa, or maculopapular cutaneous mastocytosis (MPCM), has monomorphic and polymorphic variants, depending on the size, shape, and color of the lesions. ${ }^{3}$ Monomorphic MPCM, most commonly seen in adult patients, is associated with ISM. ${ }^{3}$ Our patient had small, brownish macules on his chest and abdomen, which is compatible with 
monomorphic MPCM. The degree of skin involvement was minimal. The rash extension and density of monomorphic MPCM positively correlate with bone marrow severity, pruritus, and flushing, but not anaphylaxis. ${ }^{2,4}$ Anaphylaxis is more common in ISM without skin involvement (59\%) than in ISM with skin involvement (20\%). ${ }^{5}$ Darier's sign has a positive rate of $67 \%$ in ISM patients. ${ }^{6}$ Hyperpigmentation is sometimes difficult to discern, especially with the dark brown skin of Southeast Asians; hence, ISM might be underreported in the Asian population. There is no clear evidence that ethnic differences affect the degree and morphology of the rash in mastocytosis. An investigation of MPCM in various ethnicities found it was difficult to identify in Asians, unlike the other races studied. ${ }^{7}$ With our patient, the probable reasons for the delayed diagnosis were he had never noticed the lesions and his past physicians had not thoroughly examined his unexposed skin.

There is a firm linkage between anaphylaxis and mastocytosis. If patients present with one of these conditions, the other should be carefully investigated. Among adults with mastocytosis, $49 \%$ were found to have experienced anaphylactic episodes. ${ }^{8}$ In a study of patients with anaphylaxis, $7.7 \%$ were diagnosed with mastocytosis. ${ }^{9}$ Moreover, the prevalence of mastocytosis in patients presenting with insect sting anaphylaxis (ISA) has been reported to range from $1-7.9 \% .{ }^{10}$ The unique features of anaphylaxis in SM are male gender; triggered by an insect sting; anaphylaxis with the absence of cutaneous symptoms; and predominately cardiovascular symptoms. ${ }^{2}$ Although the patient in the current case had the classic clinical manifestations, the time between onset and diagnosis was 23 years. In contrast, the mean time lag to diagnosis for a Dutch cohort was 8 years. ${ }^{5}$ In the case of the patient described in the present study, the delay in diagnosis might have arisen from a limited awareness of primary care physicians, a lack of specialized laboratories, and a lack of consultation. The Spanish Network on Mastocytosis (REMA) developed the REMA score for mastocytosis, a clinical tool to predict mast cell clonal abnormality in bone marrow or SM. ${ }^{11}$ It draws upon 3 simple parameters (sex, clinical symptoms, and baseline serum tryptase) and has a sensitivity of $87 \%$ and specificity of $73 \% .{ }^{11}$ Our patient scored 2 points, indicating a high probability of SM. ${ }^{11}$

There is no clear evidence that some mastocytosis patients have a low or undetectable total IgE and specific $\lg \mathrm{E}^{12,13}$ This might be due to the binding of $\lg \mathrm{E}$ to excessive mast cells, resulting in lower free $\lg \mathrm{E}$ in the serum. ${ }^{12}$ The mechanism of ISA in mastocytosis is IgE and non-IgE mediated. ${ }^{12}$ It is crucial to identify the mechanism because patients diagnosed with IgE-mediated ISA need lifelong venom immunotherapy. The cutoff value for positive slgE has ranged from 0.1 to $0.35 \mathrm{kUA} / \mathrm{L}$. Michel et al. demonstrated that $8.2 \%$ of patients with mastocytosis or elevated baseline serum tryptase with a strong history of ISA would be misdiagnosed if a value of $0.35 \mathrm{kUA} / \mathrm{L}$ were used. ${ }^{13}$ Therefore, an slgE value $>0.1$ $\mathrm{kUA} / \mathrm{L}$ has been proposed as the appropriate cutoff for mastocytosis patients with ISA. ${ }^{13}$ We used 0.1 kUA/L as the cutoff for our patient.

The KIT-D816V mutation, found in $70 \%$ of adult SM patients, ${ }^{14}$ causes an increase in mast cell proliferation and survival. Apart from the KIT-D816V mutation, the TET2 mutation was found in our 
patient. The clinical manifestations associated with a TET2 mutation are female, monocytosis, and nonindolent SM. ${ }^{15}$ However, this mutation has been found in only $15 \%$ of ISM patients. ${ }^{15}$

ISM has the most favorable outcome among the SM variants. The overall survival ranges from 25.1 to 28.4 years. ${ }^{6,14}$ The chance of progression to advanced SM has been reported as $2.9 \% .{ }^{6}$ As well, the ISM predictors affecting event-free survival are an older age, male sex, low-performance status, and lymphadenopathy. ${ }^{6}$ In our case, the patient's disease remained indolent throughout the 2-year follow-up.

\section{Conclusion}

The current case highlights the importance of skin examinations and extensive workups for patients with clinical anaphylaxis. Treating physicians should carefully examine unexposed skin to check whether the patients have overlooked mastocytosis. To provide the best patient care, a multidisciplinary care team approach is required for the management of mastocytosis.

\section{List Of Abbreviations}

SM: Systemic mastocytosis

ISM: Indolent systemic mastocytosis

MPCM: Maculopapular cutaneous mastocytosis

slgE: Specific IgE

ISA: Insect sting anaphylaxis

\section{Declarations}

Ethic approval and consent to participate: An informed consent was obtained from the patient to use his data.

Consent for publication: Consent for publication was obtained from the patient.

Availability of data and materials: The data analyzed for the current case are not publicly available due the patient's privacy. Data are available from the corresponding author upon reasonable request.

Competing interests: The author declare that they have no competing interests.

Funding: No funding

Author's Contributions: CW, TS, WO provided clinical data. PS, SS reviewed skin and bone marrow pathology. CW, MS, TT drafted the manuscript. TS, WO, PS, SS revised the manuscript. All authors have read and approved the final manuscrip 
Acknowledgments: Not applicable

\section{References}

1. Horny HP, Akin C, Arber DA, Peterson LC, Tefferi A, Metcalfe DD, et al. Mastocytosis. In: Swerdlow S, Campo E, Harris N, et al., editors. WHO classification of tumours of haematopoietic and lymphoid tissues, 4th revise. Lyon, France: IARC Press; 2017.

2. Schuch A, Brockow K. Mastocytosis and Anaphylaxis. Immunol Allergy Clin North Am. 2017 Feb;37(1):153-64.

3. Hartmann K, Escribano L, Grattan C, Brockow K, Carter MC, Alvarez-Twose I, et al. Cutaneous manifestations in patients with mastocytosis: Consensus report of the European Competence Network on Mastocytosis; the American Academy of Allergy, Asthma \& Immunology; and the European Academy of Allergology and Clinical Immunology. J Allergy Clin Immunol. 2016 Jan;137(1):35-45.

4. Brockow K, Akin C, Huber M, Metcalfe DD. Assessment of the extent of cutaneous involvement in children and adults with mastocytosis: Relationship to symptomatology, tryptase levels, and bone marrow pathology. J Am Acad Dermatol. 2003 Apr;48(4):508-16.

5. Hermans MAW, Rietveld MJA, van Laar JAM, Dalm VASH, Verburg M, Pasmans SGMA, et al. Systemic mastocytosis: A cohort study on clinical characteristics of 136 patients in a large tertiary centre. Eur J Intern Med. 2016 May;30:25-30.

6. Trizuljak J, Sperr WR, Nekvindová L, Elberink HO, Gleixner KV, Gorska A, et al. Clinical features and survival of patients with indolent systemic mastocytosis defined by the updated WHO classification. Allergy. 2020 Aug;75(8):1923-34.

7. Kirshenbaum AS, Abuhay H, Bolan H, Metcalfe DD, Carter MC. Maculopapular Cutaneous Mastocytosis in a Diverse Population. J Allergy Clin Immunol Pract. 2019 Nov;7(8):2845-7.

8. Brockow K, Jofer C, Behrendt H, Ring J. Anaphylaxis in patients with mastocytosis: a study on history, clinical features and risk factors in 120 patients: Anaphylaxis in patients with mastocytosis. Allergy. 2008 Jan 4;63(2):226-32.

9. Oropeza AR, Bindslev-Jensen C, Broesby-Olsen S, Kristensen T, Møller MB, Vestergaard H, et al. Patterns of anaphylaxis after diagnostic workup: A follow-up study of 226 patients with suspected anaphylaxis. Allergy. 2017;72(12):1944-52.

10. Niedoszytko M, Bonadonna P, Elberink JNGO, Golden DBK. Epidemiology, Diagnosis, and Treatment of Hymenoptera Venom Allergy in Mastocytosis Patients. Immunol Allergy Clin North Am. 2014 May;34(2):365-81.

11. Alvarez-Twose I, González-de-Olano D, Sánchez-Muñoz L, Matito A, Jara-Acevedo M, Teodosio C, et al. Validation of the REMA Score for Predicting Mast Cell Clonality and Systemic Mastocytosis in Patients with Systemic Mast Cell Activation Symptoms. Int Arch Allergy Immunol. 2012;157(3):27580. 
12. Castells MC, Hornick JL, Akin C. Anaphylaxis After Hymenoptera Sting: Is It Venom Allergy, a Clonal Disorder, or Both? J Allergy Clin Immunol Pract. 2015 May;3(3):350-5.

13. Michel J, Brockow K, Darsow U, Ring J, Schmidt-Weber CB, Grunwald T, et al. Added sensitivity of component-resolved diagnosis in hymenoptera venom-allergic patients with elevated serum tryptase and/or mastocytosis. Allergy. 2016 May;71(5):651-60.

14. Lim K-H, Tefferi A, Lasho TL, Finke C, Patnaik M, Butterfield JH, et al. Systemic mastocytosis in 342 consecutive adults: survival studies and prognostic factors. 2009;113(23):10.

15. Tefferi A, Levine RL, Lim K-H, Abdel-Wahab O, Lasho TL, Patel J, et al. Frequent TET2 mutations in systemic mastocytosis: clinical, KITD816V and FIP1L1-PDGFRA correlates. Leukemia. 2009 May;23(5):900-4.

\section{Figures}

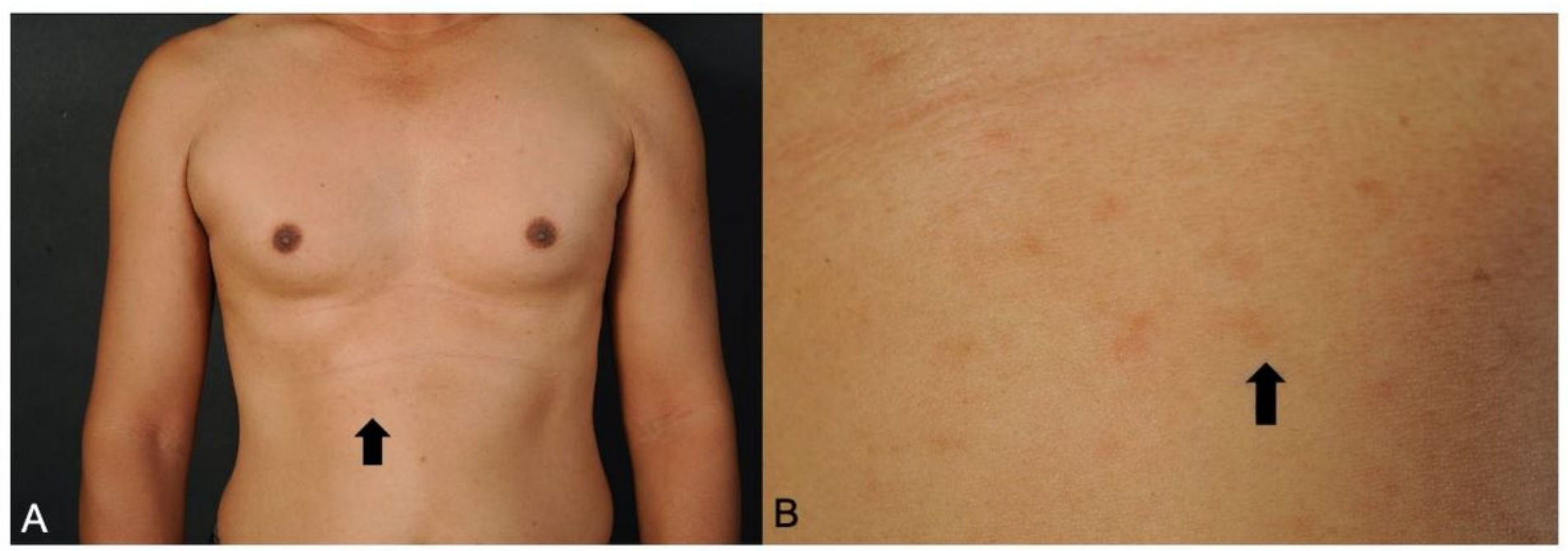

\section{Figure 1}

Multiple discrete, ill-defined, light brownish macules (arrow) on the chest and abdomen. 


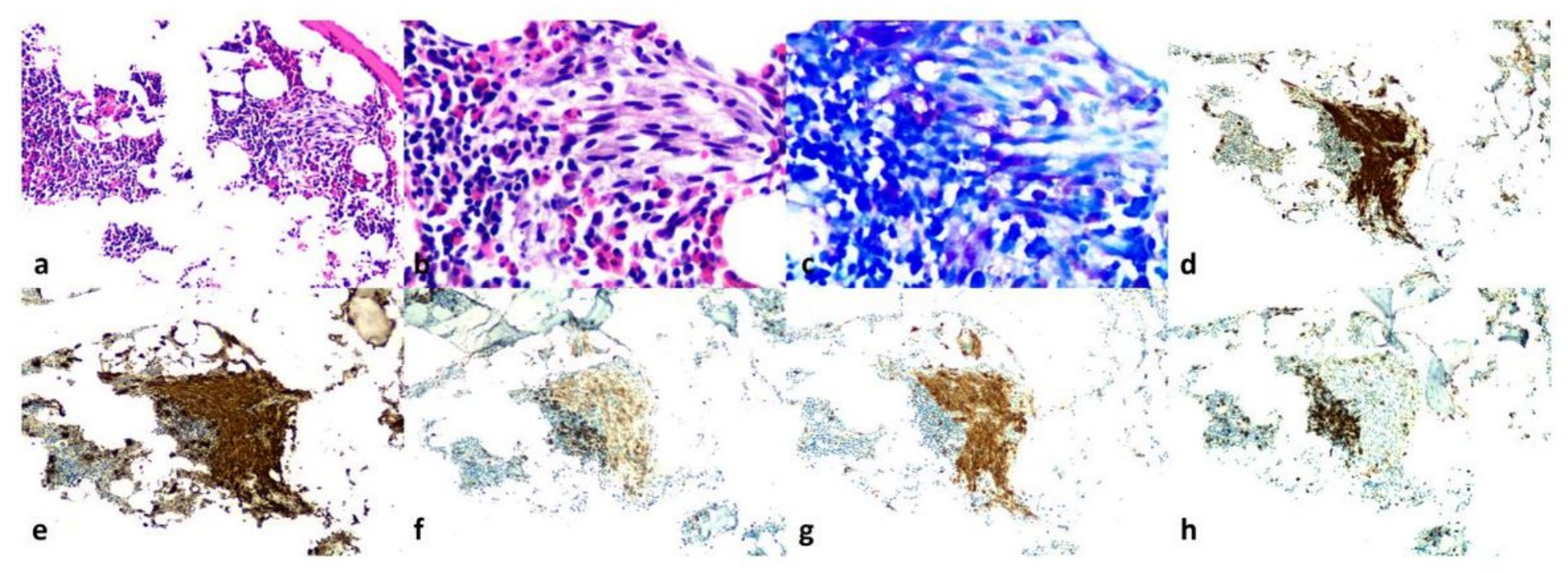

Figure 2

(a\&b) Increase in eosinophils and scattered mast cells with aggregates; many had a spindle shape, with more than 15 mast cells/high power field in aggregates (hematoxylin-eosin; original magnification $x 100$ $\& x 600$ ). (c) Some spindle-shaped mast cells containing metachromatic granules. (Giemsa; original magnification $\times 600$ ), showing positivity with (d) CD117 (CD117; original magnification $\times 100)$, (f) CD2 (CD2; original magnification $\times 100$ ), and (g) CD25 (CD25; original magnification $\times 100)$. (h) CD3 was negative in the spindle-shaped mast cells, in contrast to the adjacent small CD3+ T-cells (CD3; original magnification $\times 100$ ).

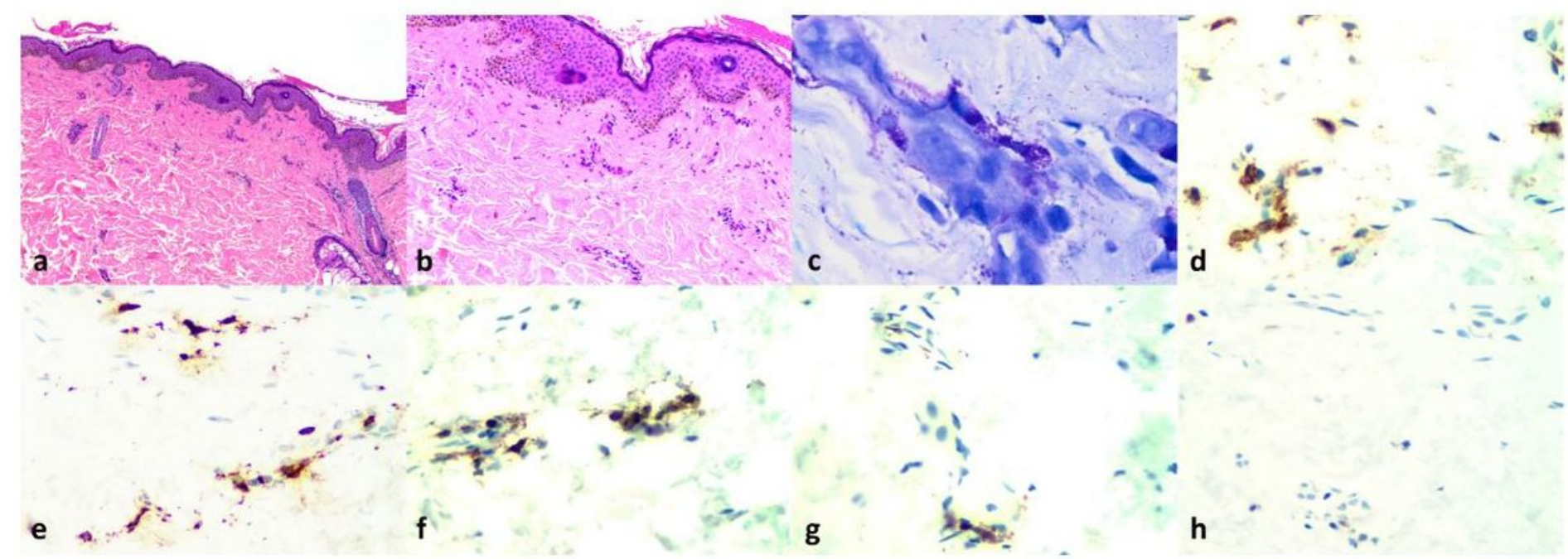

Figure 3 
(a\&b) The skin biopsy revealed superficial perivascular infiltration by mononuclear cells (hematoxylineosin; original magnification x $100 \&$ x 400). (c) Giemsa staining showed perivascular spindle cells containing metachromatic granules (Giemsa; original magnification x 1,000). (d) CD117+ mononuclear cells and spindle cells (CD117; original magnification x 400). (e) Tryptase+ mononuclear cells and spindle cells (tryptase; original magnification x 400). (f) CD2+ mononuclear cells and spindle cells (CD2; original magnification $\times 400)$. (g) Few CD25+ spindle cells (CD25; original magnification $x 400$ ). (h) No CD3 expression (CD3; original magnification $x 400$ ). 